\title{
The microwave spectrum of cyclopropane -ammonia. A novel structure for cyclopropane complexes
}

\author{
Susan E. Forest, Robert L. Kuczkowski \\ Department of Chemistry, The University of Michigan, Ann Arbor, MI 48109-1055, USA
}

Received 7 December 1993

\begin{abstract}
The microwave spectrum of the cyclopropane-ammonia $\left(\mathrm{C}_{3} \mathrm{H}_{6} \cdot{ }^{14} \mathrm{NH}_{3}\right)$ complex has been observed using a pulsed nozzle, Fourier-transform microwave spectrometer. The spectrum is characteristic of a symmetric top, $B_{0}=2668.7161$ (4), with free internal rotation of the $\mathrm{NH}_{3}$ subunit. The spectra of the $\mathrm{C}_{3} \mathrm{H}_{6} \cdot{ }^{15} \mathrm{NH}_{3}, \mathrm{C}_{3} \mathrm{D}_{6} \cdot{ }^{15} \mathrm{NH}_{3}$, and $\mathrm{C}_{3} \mathrm{H}_{6} \cdot{ }^{14} \mathrm{ND}_{3}$ isotopomers were also measured. This gives a structure in which the nitrogen of the ammonia interacts with the top of the cyclopropane ring, resulting in a stacked structure with $R_{\text {c.m. }}=3.657$ (3). The quadrupole coupling constant of the nitrogen nucleus is $e Q q=-2.509(2) \mathrm{MHz}$.
\end{abstract}

Cyclopropane (CYC) complexes, such as $\mathrm{CYC} \cdot \mathrm{HCl}[1], \mathrm{CYC} \cdot \mathrm{HF}[2], \mathrm{CYC} \cdot \mathrm{HCN}[3]$, and $\mathrm{CYC} \cdot \mathrm{H}_{2} \mathrm{O}$ [4], have been thoroughly investigated by Fourier-transform microwave (FTMW) spectroscopy in order to determine their structure. In each case the HX lies in the heavy atom plane and is hydrogen bonded to the center of one of the $\mathrm{C}-\mathrm{C}$ bonds. These studies support the Coulson and Moffitt description of cyclopropane [5] since the interaction occurs at the area of highest electron density of the ring. We have found that the complex of cyclopropane-ammonia does not conform to this pattern. Using FTMW spectroscopy we have found that it is a symmetric top, with a stacked structure, in which the lone pair of the ammonia interacts with the hydrogens above the ring. The only previous report of the $\mathrm{CYC} \cdot \mathrm{NH}_{3}$ complex was a matrix-isolation vibrational spectroscopy study which could find no evidence of complex formation in the spectra [6].

The microwave spectra of the $\mathrm{CYC} \cdot \mathrm{NH}_{3}$ isotopomers were observed using a Balle-Flygare-type FTMW spectrometer [7]. A mixture of approxi- mately $1 \%$ of each component in argon was maintained at a total pressure of 1-2 atm. Transitions found in the $\mathrm{C}_{3} \mathrm{H}_{6} \cdot{ }^{15} \mathrm{NH}_{3}$ sample showed the firstand second-order Stark shifts characteristic of a symmetric top. This led to the assignment and fitting of the transitions to Eq. (1) (below), the expression describing a symmetric top with free internal rotation about its $C_{3}$ axis $[8,9]$,

$$
\begin{aligned}
\nu= & 2 B_{0}(J+1)-4 D_{J}(J+1)^{3}-2 D_{J K}(J+1) K^{2} \\
& -2 D_{J m}(J+1) m^{2}-2 D_{J K m}(J+1) K m \\
& -2 H_{J K m}(J+1) m^{2} K^{2} .
\end{aligned}
$$

The fit for the $\mathrm{C}_{3} \mathrm{H}_{6} \cdot{ }^{15} \mathrm{NH}_{3}$ species led to assignment of the $\mathrm{C}_{3} \mathrm{H}_{6} \cdot{ }^{14} \mathrm{NH}_{3}$ transitions, found in Table 1, as well as for the $\mathrm{C}_{3} \mathrm{D}_{6} \cdot{ }^{15} \mathrm{NH}_{3}$ isotopomer. The quadrupole structure for the ${ }^{14} \mathrm{~N}$ species was difficult to decipher since the internal rotor states had splittings on the same order of magnitude as the quadrupole splittings. Only the $|m|=0,1$ states were observed; given the cold temperature of the expansion, only these states would expected to be populated. A cou- 
Table 1

Assigned transitions (center frequencies) for $\mathrm{C}_{3} \mathrm{H}_{6} \cdot{ }^{14} \mathrm{NH}_{3}$

\begin{tabular}{ccccccc}
\hline$J^{\prime}$ & $J^{\prime \prime}$ & $K$ & \multicolumn{1}{c}{$m$} & $\nu_{\text {obs }}(\mathrm{MHz})$ & $\nu_{\text {calc }}(\mathrm{MHz})$ & $\Delta \nu_{(\mathrm{obs}-\mathrm{calc})}(\mathrm{kHz})$ \\
\hline 1 & 0 & 0 & \pm 1 & 5333.583 & 5333.581 & 2.2 \\
1 & 0 & 0 & 0 & 5337.367 & 5337.366 & 1.3 \\
2 & 1 & 1 & -1 & 10666.236 & 10666.236 & 0.2 \\
2 & 1 & 0 & \pm 1 & 10666.763 & 10666.763 & 0.1 \\
2 & 1 & 1 & 0 & 10673.042 & 10673.042 & 0.2 \\
2 & 1 & 0 & 0 & 10674.330 & 10674.333 & -2.8 \\
2 & 1 & 1 & +1 & 10674.925 & 10674.926 & -0.5 \\
3 & 2 & 1 & -1 & 15998.357 & 15998.357 & -0.1 \\
3 & 2 & 0 & \pm 1 & 15999.147 & 15999.148 & -0.8 \\
3 & 2 & 1 & 0 & 16008.566 & 16008.566 & -0.1 \\
3 & 2 & 0 & 0 & 16010.504 & 16010.503 & 1.4 \\
\end{tabular}

ple of additional transitions were found within the region of the assigned lines, and although they were thought to belong to a $K=2$ transition, they were not fit due to the small number of transitions and the additional constants needed to fit those transitions. The experimentally determined spectroscopic constants for all the isotopomers may be found in Table 2 . The $\mathrm{C}_{3} \mathrm{H}_{6} \cdot{ }^{14} \mathrm{ND}_{3}$ isotopomer was also studied, but a less precise rotational constant was obtained for this species. Because of the nuclear quadrupole hyperfine interactions for the three deuteriums and the nitrogen, as well as the internal rotation splittings, the line shapes were very complicated, and no assignment was made for the hyperfine components of the different rotational transitions. An estimation for $B_{0}$ could be obtained however, based on the observed frequency regions for the $J=3-2$ and $2-1$ transitions. These transitions followed a similar frequency pattern, within and between the $J$ regions, as the other species.

The nitrogen nuclear quadruple coupling constant was determined from $\mathrm{C}_{3} \mathrm{H}_{6} \cdot{ }^{14} \mathrm{NH}_{3}$ to be $e Q q=$ -2.509 (2) $\mathrm{MHz}$, and the dipole moment of the $\mathrm{C}_{3} \mathrm{H}_{6} \cdot{ }^{15} \mathrm{NH}_{3}$ species was calculated from Stark effects for both the $J=2-1$ and $J=3-2(K=0, m=0)$ transitions, giving an observed dipole moment of $\mu=1.56(1) \mathrm{D}$ along the $a$ inertial axis.

The coordinate system describing the CYC $\cdot \mathrm{NH}_{3}$ complex can be found in Fig. 1. $R_{\text {c.m. }}$ is the distance

Table 2

Spectroscopic constants for the isotopic species of CYC $\cdot \mathrm{NH}_{3}$

\begin{tabular}{lcccc}
\hline Constants & $\mathrm{C}_{3} \mathrm{H}_{6} \cdot \mathrm{NH}_{3}$ & $\mathrm{C}_{3} \mathrm{H}_{6} \cdot{ }^{15} \mathrm{NH}_{3}$ & $\mathrm{C}_{3} \mathrm{D}_{6} \cdot{ }^{15} \mathrm{NH}_{3}$ & $\mathrm{C}_{3} \mathrm{H}_{6} \cdot{ }^{14} \mathrm{ND}_{3}{ }^{\mathrm{a}}$ \\
\hline$B_{0}(\mathrm{MHz})$ & $2668.7161(4)$ & $2582.982(2)$ & $2379.4312(8)$ & $2360(1)$ \\
$D_{J}(\mathrm{MHz})$ & $0.01661(2)$ & $0.0158(1)$ & $0.01160(5)$ & - \\
$D_{J K}(\mathrm{MHz})$ & $0.3227(3)$ & $0.328(1)$ & $0.3559(7)$ & - \\
$D_{J m}(\mathrm{MHz})$ & $1.8925(3)$ & $1.9635(2)$ & $1.6995(6)$ & - \\
$D_{J K m}(\mathrm{MHz})$ & $-1.0862(2)$ & $-1.1286(8)$ & $-1.0011(3)$ & - \\
$H_{J K m}(\mathrm{MHz})$ & $-1.2772(4)$ & $-1.334(2)$ & $-1.3610(8)$ & - \\
$\chi_{\text {aa }}(\mathrm{MHz})$ & $-2.509(2)$ & - & - & - \\
$\chi_{\text {bb }}(\mathrm{MHz})$ & $1.259(3)$ & - & 9 & - \\
$n{ }^{\mathrm{b}}$ & 12 & 12 & 1.7 & - \\
$\Delta \nu_{\text {rms }}(\mathrm{kHz})$ & 1.2 & 5.6 & &
\end{tabular}

See text for explanation about fit of the $\mathrm{C}_{3} \mathrm{H}_{6} \cdot{ }^{14} \mathrm{ND}_{3}$ isotopomer.

${ }^{b}$ Number of transitions in the fit.

${ }^{\mathrm{c}} \Delta \nu=\nu_{\mathrm{obs}}-\nu_{\text {calc- }}$ 


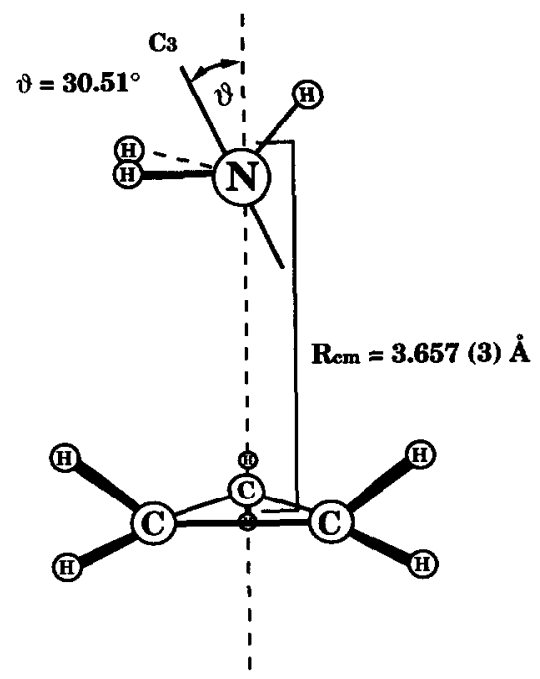

Fig. 1. Structure of the cyclopropane-ammonia complex. $\vartheta$ is the angle between the $C_{3}$ axis of ammonia and the $C_{3}$ axis of the complex, known as the effective bending angle of ammonia. $R_{\text {c.m. }}$ is the least-squares fitted distance between the centers of mass of the two monomers.

between the centers of mass of the two moieties while $\vartheta$ is the effective bending angle of $\mathrm{NH}_{3}$. If $\vartheta$ is fixed at zero and the structures of cyclopropane and ammonia remain unchanged upon complexation $[10,11], R_{\text {c.m. }}$, determined from a least-squares fit of the data, is $3.657(3) \AA$. However, the quadrupole coupling constant of the nitrogen nucleus has changed considerably in the complex $(e Q q=-2.509(2)$ $\mathrm{MHz})$ from its value in free $\mathrm{NH}_{3}\left(e Q q_{0}=\right.$ $-4.08983(2) \mathrm{MHz}$ [12]). The large change in $e Q q$ indicates the possible occurrence of large amplitude motions in this complex. To investigate these vibrational motions, we make the assumption that complex formation does not affect the electric field gradient at the nitrogen nucleus, so that the following equation can be used to estimate the value of $\left\langle\cos ^{2} \vartheta\right\rangle$ :

$e Q q=e Q q_{0}\left\langle\frac{1}{2}\left(3 \cos ^{2} \vartheta-1\right)\right\rangle$.

From this equation, $\left\langle\cos ^{2} \vartheta\right\rangle=0.7423$ implying that the effective bending angle $\vartheta$, is $30.51^{\circ}$. This can then be used to calculate $\bar{R}_{\text {c.m. }}$ in Eq. (3) (below), where $I_{\mathrm{bb}}$ and $I_{\mathrm{cc}}$ are the effective moments of inertia from each monomer obtained from refs. [10,11],

$$
\left\langle I_{\mathrm{bb}}\right\rangle^{\mathrm{CYC} \cdot \mathrm{NH}_{3}}=\mu \bar{R}_{\mathrm{c} . \mathrm{m} .}^{2}+\frac{1}{2} I_{\mathrm{bb}}^{\mathrm{NH}_{3}}\left\langle 1+\cos ^{2} \vartheta\right\rangle
$$

$$
\begin{aligned}
& +\frac{1}{2} I_{\mathrm{cc}}^{\mathrm{NH}_{3}}\left\langle\sin ^{2} \vartheta\right\rangle+\frac{1}{2} I_{\mathrm{bb}}^{\mathrm{CYC}}\left\langle 1+\cos ^{2} \gamma\right\rangle \\
& +\frac{1}{2} I_{\mathrm{cc}}^{\mathrm{CYC}}\left\langle\sin ^{2} \gamma\right\rangle .
\end{aligned}
$$

Eq. (3) also contains an angle $\gamma$, which is the bending angle of the cyclopropane. If $\gamma$ is assumed to be negligible $\left(\approx 0^{\circ}\right), \bar{R}_{\text {c.m. }}$ is calculated as $3.661 \AA$ and does not significantly change compared to the value obtained with $\vartheta=0^{\circ}\left(\bar{R}_{\text {c.m. }}=3.662 \AA\right)$.

As mentioned above, the dipole moment of the $\mathrm{C}_{3} \mathrm{H}_{6}{ }^{14} \mathrm{NH}_{3}$ species was determined from Stark effects and found to be $\mu=1.56(1) \mathrm{D}$. With the dipole moment for free $\mathrm{NH}_{3}$ which is equal to $1.47 \mathrm{D}$ [12], the induced dipole moment was calculated using the equation $\mu_{\mathrm{CYC} \cdot \mathrm{NH}_{3}}=\mu_{\text {ind }}+\mu_{\mathrm{NH}_{3}}\langle\cos \vartheta\rangle$. Setting $\langle\cos \vartheta\rangle=0.8616$ from above, $\mu_{\text {ind }}=0.29 \mathrm{D}$.

To determine whether the umbrella of ammonia was pointing up, away from the cyclopropane ring or down, towards the cyclopropane ring, it was necessary to study the $\mathrm{C}_{3} \mathrm{H}_{6} \cdot{ }^{14} \mathrm{ND}_{3}$ isotopomer. Predictions of the $B_{0}$ rotational constant for the two structures (umbrella up and umbrella down) were 2350 and $2453 \mathrm{MHz}$, respectively. The observed $B_{0}$ value of $2360 \mathrm{MHz}$ was consistent with the ammonia umbrella pointed up, away from the cyclopropane ring, leaving the lone pair to interact with the ring.

This configuration was also consistent with the electrostatic model of Buckingham and Fowler [13]. The electrostatic interaction energy was calculated for both the umbrella up and umbrella down structures at the observed $R_{\text {c.m. }}$, for two different conformations of the ammonia about the $C_{3}$ axis. One conformer has the hydrogens of ammonia eclipsed with respect to the carbons of the cyclopropane ring, and the other conformation has the hydrogens staggered. The umbrella up structure has a favorable interaction energy for both the eclipsed $\left(E_{\mathrm{e}}\right)$ and staggered $\left(E_{\mathrm{s}}\right)$ configurations $\left(E_{\mathrm{e}}=-0.118 \mathrm{kcal} / \mathrm{mol}, E_{\mathrm{s}}=-0.250 \mathrm{kcal} /\right.$ mol) unlike the umbrella down structure where both conformations have energies that are not stable $\left(E_{\mathrm{e}}=0.563 \mathrm{kcal} / \mathrm{mol}, E_{\mathrm{s}}=0.863 \mathrm{kcal} / \mathrm{mol}\right)$. Of course, the spectral data cannot distinguish between the staggered and eclipsed conformations. It may be notable that the interaction energies are small, suggesting a weak interaction. This is also suggested from the pseudodiatomic model which gives a stretching force constant of $0.036 \mathrm{mdyn} / \AA$ and a binding energy of $340 \mathrm{~cm}^{-1}$ for a Lennard-Jones potential. For comparison, the four complexes of cyclopropane with 
$\mathrm{HX}$ and $\mathrm{H}_{2} \mathrm{O}$ in the introduction have binding energies ranging from $540-830 \mathrm{~cm}^{-1}$ when calculated in a similar manner.

It is also interesting that the Buckingham and Fowler model gives an energy difference between the staggered and the eclipsed conformations $\left(E_{\mathrm{s}}-E_{\mathrm{e}}\right)$ for the umbrella up structure of $0.132 \mathrm{kcal} / \mathrm{mol}$, or approximately $46 \mathrm{~cm}^{-1}$. This suggests a non-zero barrier to internal rotation. This is within the upper bound for an estimated internal rotation barrier from the spectral data. The latter estimate was made using a PAM internal rotation program [14] to fit the observed transitions to rotational and distortion constants, given a certain barrier height. Reasonable fits of the spectra could be obtained for barriers up to 60 $\mathrm{cm}^{-1}$. Therefore, the frequency data are consistent with an internal rotation barrier for $\mathrm{CYC} \cdot \mathrm{NH}_{3}$ of $0 \leqslant V \leqslant 60 \mathrm{~cm}^{-1}$.

It is clear that the configuration found here is different from previous cyclopropane complexes with acids and water. Comparing this system to other ammonia complexes is a bit morc complicated, as there are many ammonia complexes which have been studied, yet none quite like this one where the ammonia is interacting with a saturated hydrocarbon. A possible comparison could be with the T-shaped complex of $\mathrm{NH}_{3} \cdot \mathrm{NCCN}$ [15], with $R_{\text {c.m. }}=3.13 \AA$; this value is substantially smaller than that determined for the $\mathrm{CYC} \cdot \mathrm{NH}_{3}$ complex $\left(R_{\mathrm{c} . \mathrm{m} .}=3.65 \AA\right)$. Another comparison is to the symmetric top $\mathrm{CF}_{3} \mathrm{H} \cdot \mathrm{NH}_{3}$ [8], where the $R_{\text {c.m. }}$ distance is $3.84 \AA$ and free or nearly free internal rotation also occurs. These complexes, however, are not really close structural analogues to $\mathrm{CYC} \cdot \mathrm{NH}_{3}$ where the ammonia is sitting directly above the center of the ring, presumably in the best position to enhance the interaction between the lone pair and the positive region above the carbon plane.

In this complex, cyclopropane does not use its wellknown "bent bonds" to form a complex, but rather utilizes a part of the molecule that is more hydrogen rich. It will be interesting to learn whether this presages the interaction of ammonia with other satu- rated hydrocarbons, notably propane. There are no other examples in the literature of gas phase complexes in which cyclopropane is the hydrogen donor and/or where ammonia is interacting with a saturated, non-substituted hydrocarbon without an acidic hydrogen.

This work was supported by the National Science Foundation. An allotment of computer time for calculations at the San Diego Supercomputer is gratefully acknowledged. SEF also wishes to acknowledge Dr. Amine Taleb-Bendiab for help in running the internal rotation program.

\section{References}

[1] A.C. Legon, P.D. Aldrich and W.H. Flygare, J. Am. Chem. Soc. 104 (1982) 1486.

[2] L.W. Buxton, P.D. Aldrich, J.A. Shea, A.C. Legon and W.H. Flygare, J. Chem. Phys. 75 (1981) 2681.

[3] S.G. Kukolich, J. Chem. Phys. 78 (1983) 4832.

[4] A.M. Andrews, K.W. Hillig II and R.L. Kuczkowski, J. Am. Chem. Soc. 144 (1992) 6765.

[5] C.A. Coulson and W.E. Moffitt, Phil. Mag. 40 (1949) 1.

[6] A.J. Barnes and S.L. Paulson, Chem. Phys. Letters 99 (1983) 326.

[7] K.W. Hillig II, J. Matos, A. Scioly and R.L. Kuczkowski, Chem. Phys. Letters 133 (1987) 359; T.J. Balle and W.H. Flygare, Rev. Sci. Instr. 52 (1981) 33.

[8] G.T. Fraser, F.J. Lovas, R.D. Suenram, D.D. Nelson Jr. and W. Klemperer, J. Chem. Phys. 84 (1986) 5983.

[9] W.H. Kirchhoff and D.R. Lide Jr., J. Chem. Phys. 43 (1965) 2203.

[10] Y. Endo, M.C. Chang and E.H. Hirota, J. Mol. Spectry. 126 (1987) 63.

[11] M.D. Harmony, V.W. Laurie, R.L. Kuczkowski, R.H. Schwendeman, D.A. Ramsay, F.J. Lovas, W.J. Lafferty and A.G. Maki, J. Phys. Chem. Ref. Data 8 (1979) 647.

[12] M.D. Marshall and J.S. Muenter, J. Mol. Spectry. 85 (1981) 322.

[13] A.D. Buckingham and P.W. Fowler, Can. J. Chem. 63 (1985) 2018.

[14] A. Taleb-Bendiab, K.W. Hillig II and R.L. Kuczkowski, J. Chem. Phys. 97 (1992) 2996.

[15] I.I. Suni, S. Lee and W. Klemperer, J. Phys. Chem. 95 (1991) 2859. 